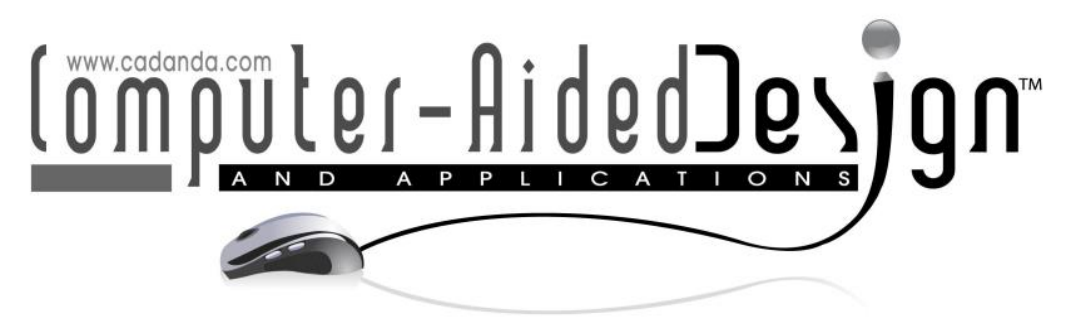

\title{
Design and Simulation of Computer Aided Chinese Vocabulary Evaluation System
}

\author{
Quanyue Wang1 (D), Sisi Zhang² (D) and Weichao Liu³ (iD) \\ ${ }^{1}$ School of Chinese Culture and Communication, Beijing International Studies University, Beijing \\ 100024, China, wangquanyue@bisu.edu.cn \\ ${ }^{2}$ Department of Automation, College of Information Science and Engineering, China University \\ of Petroleum Beijing Campus (CUP), Beijing 102249, China, 18811170278@163.com \\ ${ }^{3}$ Academic Affairs Office, China University of Petroleum Beijing Campus (CUP), Beijing 102249, \\ China, weichao828@163.com
}

Corresponding author: Quanyue Wang, wangquanyue@bisu.edu.cn

\begin{abstract}
This article first explains the research background of computer-aided Chinese vocabulary assessment. Vocabulary assessment occupies an important position in Chinese learning. Computer-aided assessment has advantages in statistical analysis and organization and management. Subsequently, the development of Chinese vocabulary assessment and computer-assisted Chinese assessment was reviewed, and the design scheme of computer-assisted Chinese vocabulary assessment system was constructed. The functional framework, assessment methods, and feedback of the assessment system were explained. Finally, this paper verifies the function of the system through experimental investigation.
\end{abstract}

Keywords: Computer-aided Chinese vocabulary test; Evaluation feedback DOI: https://doi.org/10.14733/cadaps.2021.S3.1-11

\section{INTRODUCTION}

Vocabulary is closely related to Chinese skills. The mastery of Chinese vocabulary affects the comprehension of listening and reading, the richness of speaking and writing of Chinese learners [1]. The importance of vocabulary is deeply rooted in the hearts of the people, and vocabulary assessment can provide teachers and students feedback information to improve the quality of Chinese learning. Therefore, whether it is language teaching research experts, teachers or students, all attach great importance to vocabulary testing [2]. For teachers, testing the learners' vocabulary level before teaching helps teachers determine the progress of vocabulary learning and the focus of vocabulary teaching, so as to adopt targeted teaching strategies to achieve the 
teaching goals. Vocabulary test after teaching can test the results of teaching and adjust teaching activities; for learners, vocabulary test can help learners fully understand their Chinese proficiency, so as to choose targeted learning content and learning strategies, and vocabulary. The test allows learners to quickly and conveniently understand their own learning progress, and intuitively watching their own progress every day will stimulate learners' enthusiasm for learning and increase their interest in Chinese learning; for researchers, Chinese vocabulary testing can be used experimental research to verify the effects of different vocabulary teaching (learning) methods and teaching (learning) strategies.

In the organization and management of evaluation, Lyu et al. [3] said computer-aided evaluation is not restricted by time and space. Through the electronic transmission of test materials, the large-scale group assessment is more material-saving, and the organization is relatively simple. The analysis, retention and query of test papers after the test are very convenient and efficient. In addition, building a large and orderly question bank through a computer is more convenient and more scientific than manual examination. In the correction and statistics of the results, the traditional evaluation requires a lot of time and energy for teachers, while the automatic scoring by computers is fast and accurate. At the same time, it avoids errors in manual scoring due to human factors and saves a lot of manpower and material resources. In terms of evaluation feedback, $\mathrm{Li}$ et al. [4] said computer-aided evaluation can provide instant feedback. When a learner is performing an exercise or test, he can get systematic feedback as long as he enters the data, and he knows what is right and what he has mastered in real time. It is convenient for the learner to understand his learning progress at any time, and it has a great advantage over traditional assessment in formative evaluation. Quick and instant access to personalized feedback is one of the biggest advantages of computer-aided assessment. In addition, the feedback quality of traditional assessments depends on the level of teachers, while the computer-assisted Chinese assessment system has a built-in feedback system, so that every learner can get feedback of the same standard [5]. Finally, people realize that non-project factors in the evaluation process affect the evaluation results. The computer can track the entire evaluation process well and collect evaluation data, such as the time taken by the subjects to test, the order and number of questions, whether the answers have been changed, etc. These factors are incorporated into the basis of the evaluation to improve the validity of the evaluation. It can be seen that computer-assisted assessment has huge advantages over traditional assessment, and it is the future development direction of assessment, and it is also a topic of focus in the research field of Chinese assessment.

In this paper, vocabulary assessment occupies an important position in Chinese learning. However, traditional vocabulary assessment has many limitations in terms of feedback time, efficiency, quality and effectiveness. Computer-aided assessment has its advantages in statistical analysis, organization and management. The above advantages have become an effective means to make up for the lack of traditional vocabulary assessment. Therefore, this article intends to conduct related research on computer-aided Chinese vocabulary assessment.

\section{CHINESE VOCABULARY EVALUATION SYSTEM DESIGN}

\subsection{Design Goals}

Only by fully understanding the needs of system users and under the guidance of clear goals can we design and establish a practical and valuable vocabulary assessment system. From the above review, we can know that the current vocabulary assessment has the following shortcomings: First, the amount of feedback received by teachers and students is small, and the feedback effect is not good. The result of the traditional vocabulary assessment method is only a score, which can only understand the general level of the student, and the mastery of the specific knowledge behind the score is not known; second, the efficiency of the traditional vocabulary assessment is low, stated by $\mathrm{Wu}$ et,al[6]. Designing vocabulary assessment items, reviewing test questions, statistics and

Computer-Aided Design \& Applications, 18(S3), 2021, 1-11

(C) 2021 CAD Solutions, LLC, http://www.cad-journal.net 
analyzing assessment results often consume a lot of energy for teachers, and it is difficult for teachers to give immediate feedback to students. Therefore, there is an urgent need for a Chinese vocabulary assessment system with automatic review and scientific feedback functions to reduce the burden on teachers, improve teaching efficiency and effectiveness, and meet the needs of further development of Chinese international education [7].

The system is expected to achieve the following goals:

(1) The interface is friendly, simple and beautiful, and easy to operate.

(2) The system has an automatic review function, which can provide immediate feedback on the evaluation results.

(3) The system can feedback the mastery of individual vocabulary knowledge and overall vocabulary knowledge.

(4) Students can easily access the evaluation results, analysis of wrong questions and related suggestions.

(5) The system can count performance analysis indicators such as the highest score, lowest score, average score and standard deviation, and provide feedback for teachers and administrator users.

(6) Teachers can create their own question bank, add, delete, and modify questions; administrators can add, delete, and modify the system, built-in test question bank.

(7) The administrator can manage and maintain the system, and can view and modify the information of teachers, students and question banks.

(8) The functions of each module of the system can be expanded, and other types of questions can be added as required, with good expandability. After being expanded, it can meet the needs of other types of Chinese test besides Chinese vocabulary test.

\subsection{System Framework}

There are three types of users of the Chinese vocabulary assessment system: students, teachers and system administrators. By using the evaluation system, students can conduct self-tests and obtain corresponding learning feedback, thereby improving their learning. Through the system, teachers can understand the vocabulary mastery of the students taught, create their own question bank, organize students to take exams, and improve their work efficiency. The administrator has the highest authority to manage and maintain students, teachers and question banks, as stated by Zhang et al. [8]. The functional framework of the Chinese vocabulary assessment system is shown in Figure 1. Through the Chinese language assessment, a formative evaluation based on the learning process and a summary evaluation based on the results of the learning phase are made to examine the true state of knowledge and language ability of the students. Jianwei et al. [9] stated that the purpose is to scientifically, comprehensively, fairly and accurately measure the students' knowledge. The level of mastery and true language ability, to detect the learning effect of relevant knowledge, to understand and master the deficiencies and obstacles of students in learning; to use the backwash effect of the evaluation results, on the one hand, to give learners the correct language output and understanding affirmatively, strengthen the correct language rules in the knowledge structure and encourage learners to further Chinese learning. On the one hand, it points out the problems and obstacles in the learners' Chinese learning, suppresses and corrects the learners' incorrect knowledge schema, and helps the learners to go smoothly in time complete the acquisition of Chinese knowledge and skills; it is to provide relevant reference basis and standards to facilitate the screening and selection of students.

For Chinese teaching, the purpose of Chinese assessment is to help teachers understand and master the knowledge structure and skill level of students, check the teaching effects of relevant knowledge content, adjust teaching progress in time according to the assessment results, improve teaching methods, and implement relevant teaching remedial measures. It is to monitor and evaluate teaching objectives, and provide scientific and objective reference standards and basis for the formulation of syllabus and teaching plan. 


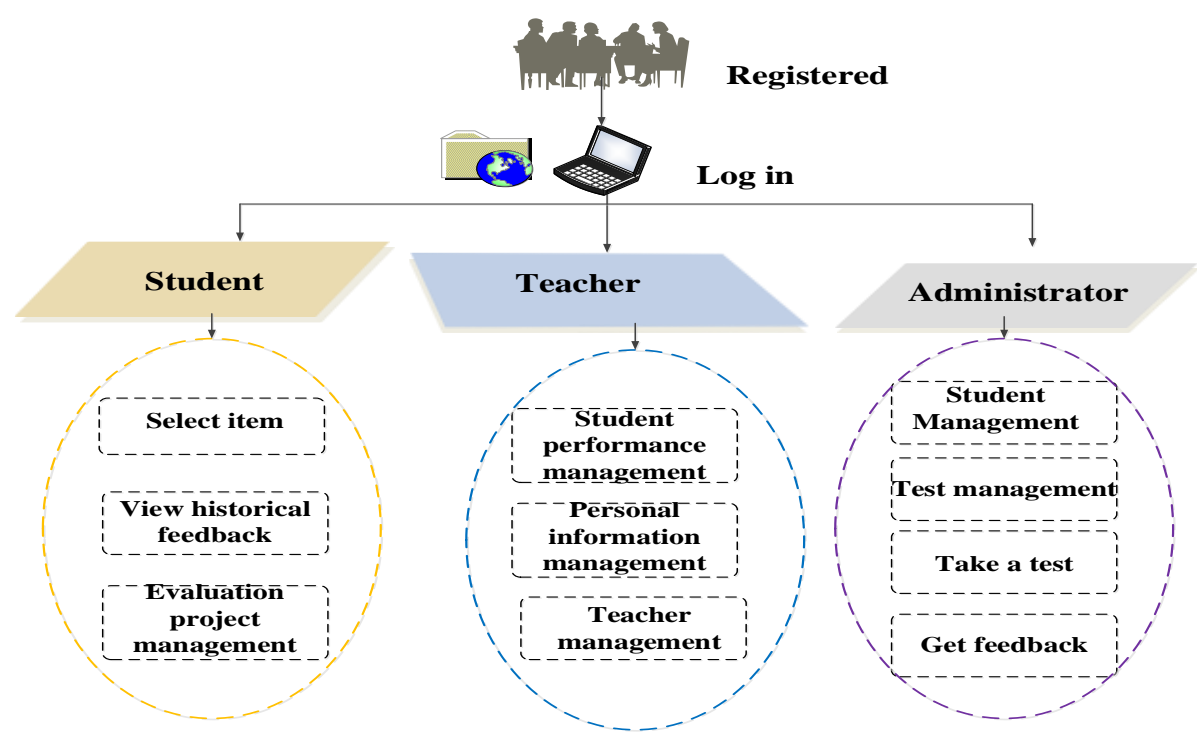

Figure 1: System function framework.

In order to achieve the above functions, the system will be divided into the following five modules: registration and login module, test module, review module, feedback module and management module, as shown in Figure 2.

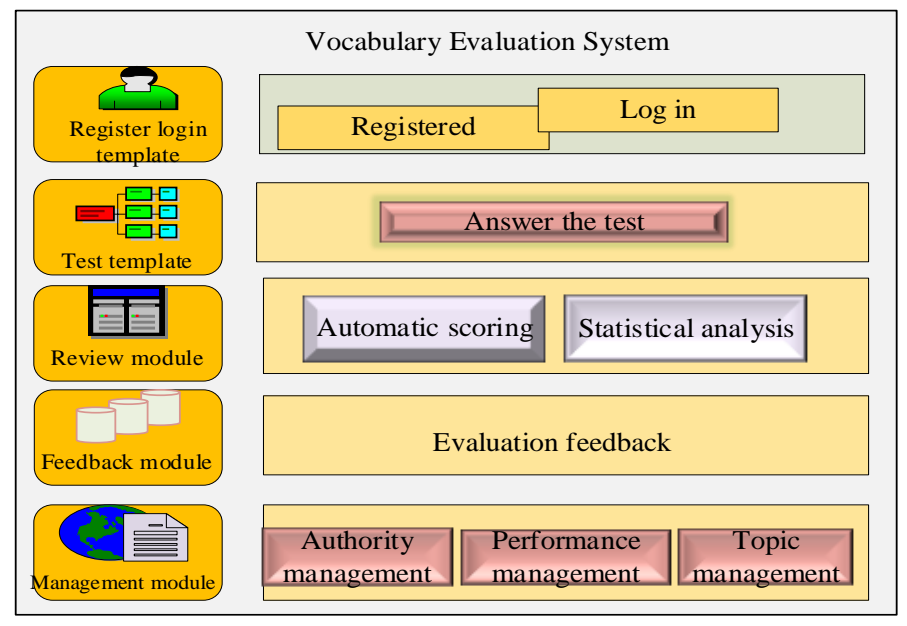

Figure 2: System components.

\subsection{Evaluation Process}

In order to adapt to the development trend of computer-assisted assessment and enable learners to use the vocabulary assessment system for Chinese learning conveniently, this system adopts a browser-based B/S (Browser/Server) structure. As long as learners can access the Internet, they can use this system. system.

The B/S structure is divided into three parts: browser, server and database (also called presentation layer, function layer and data layer). Browser is a user-oriented interface and a 
window for information interaction. The server layer is composed of Web pages. The server layer is the core part of the realization of system functions, according to Ravi et al.[10]. Data processing and statistical analysis functions are concentrated in this part. The database mainly stores user information, topic information, and data generated during testing.The process of computer-aided vocabulary assessment is shown in the Figure 3 below:

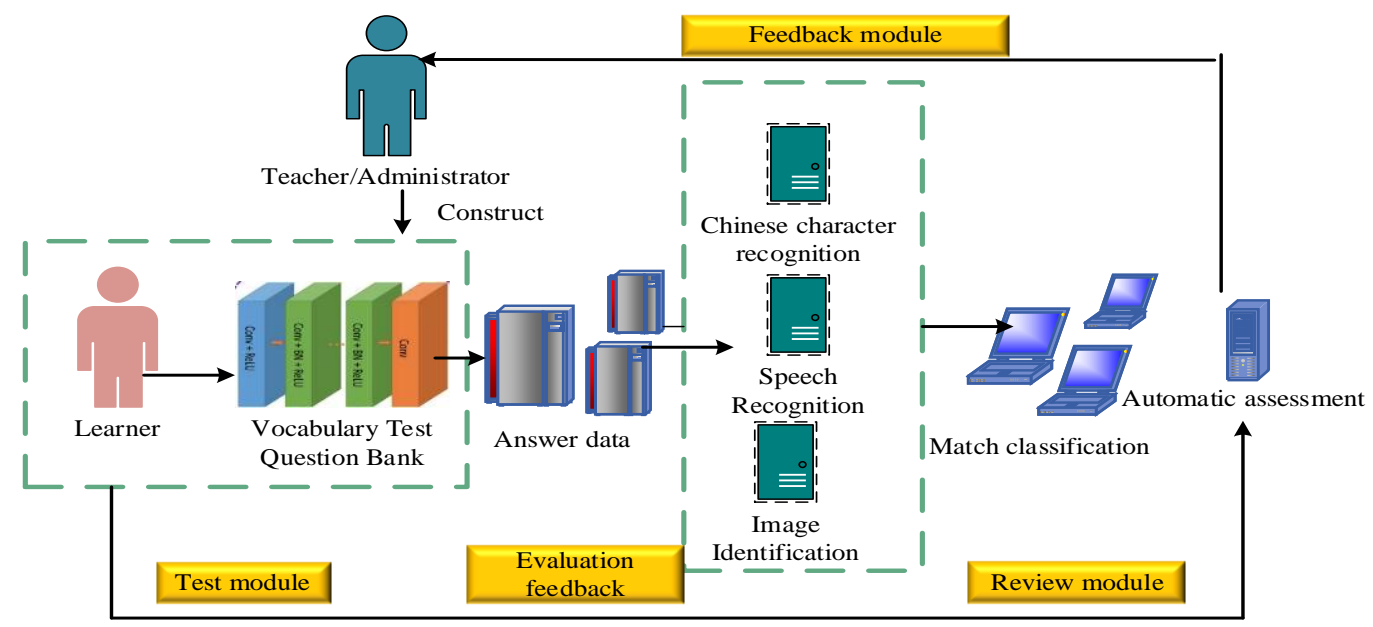

Figure 3: Computer-aided vocabulary assessment process.

The process of computer-aided vocabulary assessment can be divided into the following parts:

2.3.1 Construction of vocabulary assessment resources (question bank)

Use words, sounds, charts, images, and videos, multimedia technologies such as animation and animation are used to construct vocabulary assessment resources, which are presented to the assessors in a more humane, close to the physical and psychological needs of learners, and build a good assessment environment close to the real language communication situation for learners, stated by Yang, L et al [9].

2.3.2 Recognition of response data

Through natural language processing technology, including Chinese character, speech recognition and other data processing technology, the data input by the learner is recognized and converted into a form that the computer can understand.

2.3.3 Automated evaluation

Automated assessment is also called computer automated scoring (CAS), refers to the process of computer simulating manual scoring to score questions (including objective questions and open questions), skills, operations, and performance activities.

2.3.4 The construction of feedback content

Feedback content can guide students' learning, help teachers improve teaching and adjust teaching goals. The evaluation results are processed through multimedia technology, and the evaluation results are presented to users in a variety of targeted forms. The design and construction of feedback content is the research focus of computer-aided vocabulary assessment.

\section{INVESTIGATION AND ANALYSIS OF THE APPLICATION OF CHINESE VOCABULARY ASSESSMENT SYSTEM}

\subsection{Purpose and Object of Investigation}


By investigating the use of the computer-assisted Chinese vocabulary assessment system, testing the effectiveness of the system, collecting the use evaluation of students and teachers, and using this as a basis for further improvement of the assessment system, it provides a basis for the follow-up research of computer-aided assessment. For reference, further promote the development of Chinese international education. With the rapid development of information technology, natural language processing technology and language measurement technology, computer-assisted Chinese assessment has become an important means to improve the efficiency and scientific degree of assessment. Computers are used in automatic scoring, test paper composition, project analysis, test paper analysis, and test paper management. And examination management plays an important role. This article analyzes the theoretical basis of computer-aided evaluation: classical measurement theory, item response theory, and cognitive diagnosis theory. The latter two are the current research hotspots, but the domestic research results are not very rich, and they are still in the stage of exploration. The evaluation system based on item response theory and cognitive diagnosis theory has higher technical requirements and complex mathematical models. Therefore, a more mature classical measurement theory is a more feasible choice. In the teaching of Chinese as a second language, some assessment systems have also emerged. However, analyzing these systems shows that these systems can make up for some of the limitations of traditional assessment, but they are not detailed enough in feedback, lack statistical analysis functions, and lack a specialized assessment system for certain skills or knowledge of Chinese. The target users of this system are college students and adult Chinese learners. Therefore, this survey selected 30 international students and 3 teachers from the Department of Education of the college to conduct the survey.

\subsection{Investigation Method}

This survey adopted two methods: questionnaire survey and interview. 30 students who participated in the system test were asked to complete the questionnaire, and interviewed teachers and some students. The students' interviews are explained in the analysis of the questionnaire survey results, and the teacher's interview results are presented in the appendix in the form of a report. The data obtained from the survey is statistically analyzed with the help of Excel software.

\subsection{Survey Content}

The survey content mainly covers the following aspects:

(1) The design level of the system, including operation and interface design.

(2) The feedback function of the system, including the user's evaluation of the feedback form, time and content.

(3) The quality and quantity of system test questions.

(4) The user's recognition of the system, including the system's promotion of Chinese learning, the system's information feedback effect and the user's willingness to continue using it.

\section{SURVEY RESULTS AND ANALYSIS}

\subsection{The Design Level of the System}

The design level of the system is mainly investigated from the operation of answering questions and the design of the interface. The results of students' feedback are as shown in Figure 4.

$56.4 \%$ of students think that the operation of the system is very convenient, and $34.2 \%$ of students think that the operation is convenient. The combined ratio of these two items exceeds $90 \%$, which shows that students have a high degree of recognition for the convenience of the vocabulary assessment system. Convenient operation is the advantage of computer-assisted assessment. The answer can be completed with the click of the mouse. After answering the 
question, it will automatically enter the next link. After the test, the result will be automatically fed back to the learner. The degree of automation is better.

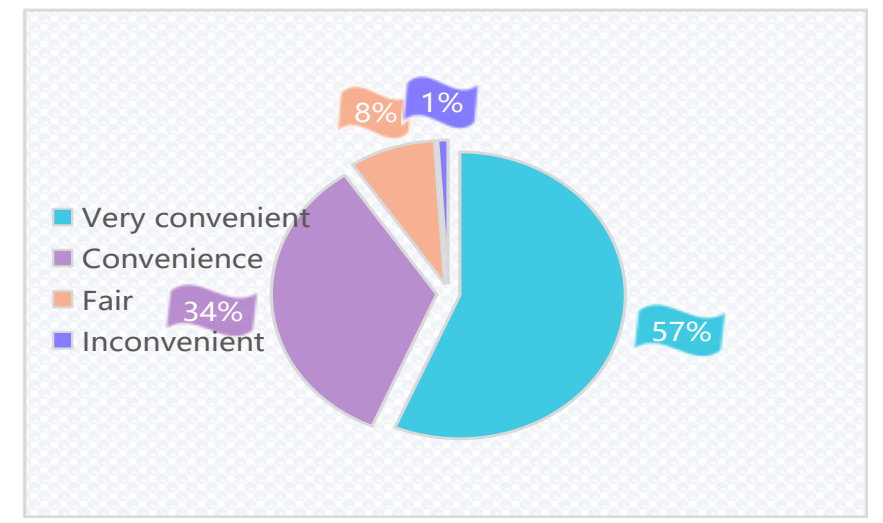

Figure 4: Convenience of system operation.

\subsection{System Feedback Function}

The feedback function is the characteristic of the system and the focus of the investigation. The questionnaire surveyed the form, time and content of the feedback. The results are shown in Figure 5.

The feedback forms of the vocabulary system include text, graphics, charts, sounds and FLASH stroke order animation, which are rich in forms. Students also have a high evaluation of the feedback form. $61.4 \%$ of the students think that the feedback form is very practical, and $24.2 \%$ of the students think the feedback form is more practical. The proportion of these two items exceeds $90 \%$. However, the feedback form of this system is generally relatively simple and more suitable for adult Chinese learners. For younger students, more improvements are needed. The feedback content of the vocabulary system includes the overall vocabulary mastery, ranking and the pronunciation, shape, meaning and use of each vocabulary. $56.3 \%$ of users think that the content of feedback is very effective for learning Chinese, and $42.1 \%$ of students think that the content of feedback is more effective. This shows that the content of system feedback is more comprehensive and learners can get the feedback information they need.

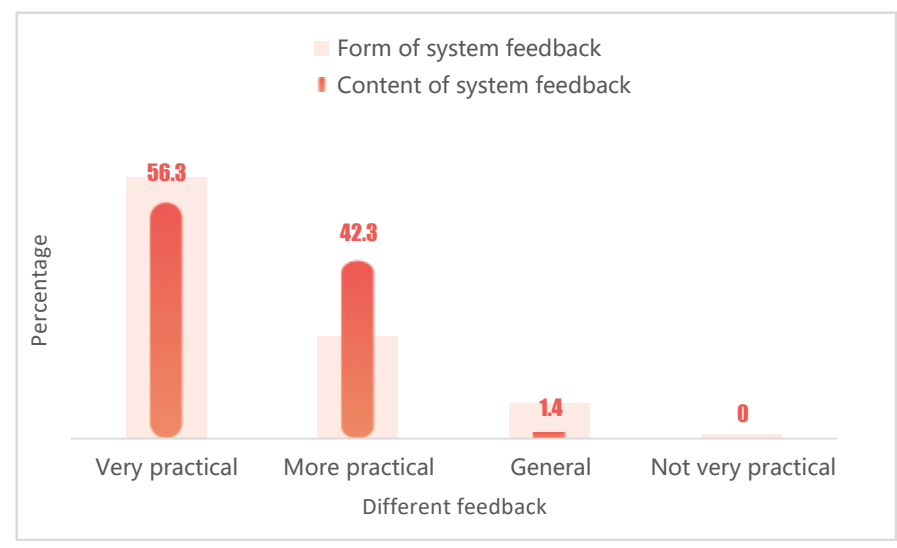

Figure 5: The form and content of system feedback. 


\subsection{The Number of Test Words}

This survey item is set up to understand the appropriate range of the number of tested words. In the first volume of "Chinese Course", there are about 15 new words in each lesson, the first volume is 25-30 new words in each lesson, and the second volume has more than 35 new words in each lesson, the most. There are 40 new words in one lesson, and the number of words in each lesson of "Chinese Course" is relatively large. There are 30 words and 60 questions in this test. The feedback from students is shown in Figure 6:

Test words Questions

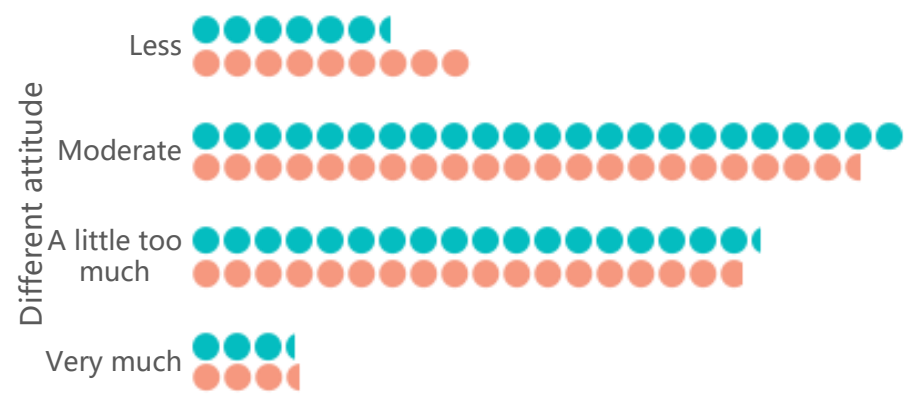

Percentage

Figure 6: The number of test questions and the number of test words.

The survey shows that $6.7 \%$ of students think that the number of questions is very large, $34.3 \%$ of students think that the number of questions is a little too much, $41.6 \%$ of students think that the number of questions is moderate, but $17.4 \%$ of students think that the number of questions is small. In terms of the overall situation, the test questions this time are medium to large. Regarding how many words need to be tested in a test, $6.4 \%$ of the students think $10-15$ words are suitable, $35.4 \%$ of the students think $16-25$ are suitable, $44.3 \%$ of the students think $26-30$ are more suitable, and $12.4 \%$ Of students think 30 or more are acceptable. After checking their Chinese proficiency through the system, it is found that the number has a greater relationship with the level of Chinese proficiency. Students with high learning levels tend to have more numbers, while students with average Chinese proficiency hope not to test too much in one test. On the whole, the 16-30 interval is more appropriate. In the future system improvement, the function of selection can be added, and students can choose the words and the number they need to test, which is more targeted.

\subsection{System Recognition}

The questionnaire surveys the students' recognition of the system from three aspects: the system' $s$ role in promoting Chinese learning, the role in helping students understand their own situation, and the students' willingness to continue using the system. The survey uses Liken's 5-level scale to set up questions, which are completely agree, relatively agree, fair, disagree, and completely disagree, and mark them as 1-5 points. 5 is the perfect score, 1 is the lowest score, and a score of 3 indicates that the learner is neutral with the system and does not like or dislike it. The results of the investigation are shown in Table 1 and Figure 7: 


\begin{tabular}{ccccccc}
\hline Item & 1 & 2 & 3 & 4 & 5 & Total scores \\
Promotion of Chinese learning & 0 & 0 & 2 & 12 & 16 & 4.3 \\
Role in understanding learning & 0 & 2 & 4 & 16 & 11 & 4.2 \\
Willingness to continue using the system & 0 & 2 & 3 & 11 & 13 & 4.5 \\
\hline
\end{tabular}

Table 1: System recognition level.

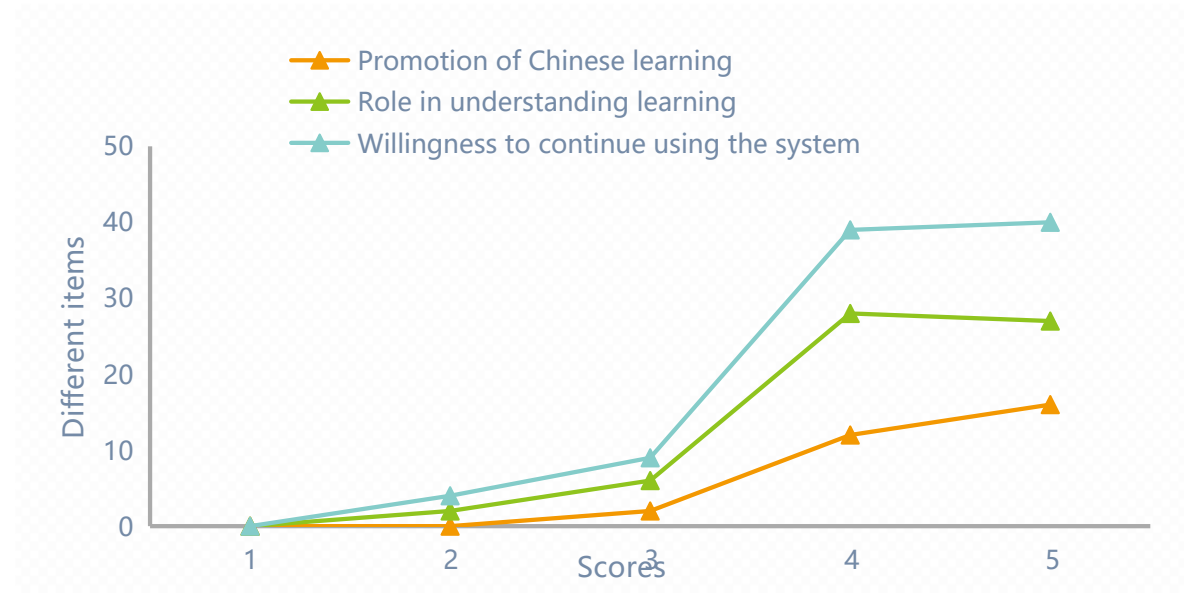

Figure 7: The scores of system recognition level.

On the whole, students recognized the Chinese vocabulary assessment system, with scores exceeding 4.2 points, indicating that students have a positive attitude towards the function of the system. The score for the option "Promoting Chinese Learning" reached 4.5, indicating that students agree with the statement "Chinese vocabulary assessment system can help me learn Chinese". Through the questionnaire survey, we have a better understanding of learners' usage and feelings, and also verified the effectiveness of the computer-assisted Chinese vocabulary assessment system. The evaluation system has been well received in terms of design (interface, operation), feedback function (form, time, form, content and effect). There are too many questions and words in the test, and the reasonable range of the number of words is 16-25. Students have a high degree of recognition of the evaluation system, and the system basically achieved the set goals and achieved good results.

At the same time, some problems were discovered in the investigation, such as the system's failure to return data in time during multi-user access, and some negligence and deficiencies in the design, which need to be improved and perfected in the next step of the research work.

\section{DISCUSSION}

This research mainly builds a test platform based on examples of Chinese vocabulary test and assessment, which lays the foundation for subsequent research. However, the construction and improvement of the computer-assisted Chinese vocabulary assessment system is a long-term process, which requires the cooperation of foreign Chinese teachers, computer technicians and users. At present, this research has the following shortcomings: the content and form of Chinese vocabulary assessment have not been verified by scientific experiments, and the subjectivity is strong; the form of the test questions is still not comprehensive due to technical limitations; pronunciation, writing and subjective questions cannot be automatically scored. In general, many aspects of the system still need to be improved.

(1) Enrich the content of the question bank 
At present, there are not many question banks in the system, which are not enough to meet the needs of teachers and students. This requires full cooperation with experienced teachers of Chinese as a foreign language and Chinese assessment experts to design and enter more high-quality questions for the system. Scientific and high-quality question bank can maximize the value of the system.

(2) Optimize the volume strategy

The current system only has manual test paper composition mode: first set the frame and basic information of the test paper, and then manually enter the questions to complete the test paper addition, the whole process is operated by the user. However, such a model cannot guarantee the scientific nature of the test paper. In the future design, consider adding test paper strategies including difficulty ratio, time ratio, and question type ratio. With the enrichment of the question bank and the improvement of the test paper strategy, the automatic test paper module can be added to further reduce the burden on teachers.

(3) Improve the system interface

The interface is the most direct module presented to the user by the system, which affects the user's experience. A good interface will make the user easy to accept and happy to use the system. The current system interface is relatively rough and needs further improvement. It needs to be optimized for users of different Chinese proficiency and different ages to meet the needs of users.

(4) Build a humane evaluation environment

This system uses text, sound, charts and other media forms to create an environment for evaluation tasks. Although it has a certain positive effect, it fails to fully utilize the advantages of multimedia. In the use of multimedia technology and the construction of the evaluation environment, more investigations and more innovations should be done to build a good evaluation environment close to the real language communication situation for learners.

\section{CONCLUSION}

This research has designed and developed a computer-assisted Chinese vocabulary assessment system for Chinese teaching, and has made useful explorations in Chinese vocabulary assessment and computer-assisted Chinese assessment. This paper analyzes the assessment, vocabulary assessment and computer-assisted assessment of Chinese as a second language teaching. The current situation and development of Chinese language assessment, discusses the necessity and feasibility of building a computer-aided Chinese vocabulary assessment system. This article clarifies the needs of system users, puts forward the goals of system design, system framework, modules and the functions of each module, and initially forms the overall plan of the computer-aided Chinese vocabulary assessment system. According to the needs of teachers and students, targeted feedback projects and corresponding forms for teachers and students are designed to construct a basic usable framework. According to the system design plan, computer technology is used to basically realize the system's registration and login, management, testing, review and feedback functions, and achieve the set goals. This paper investigates the application of the system, collects the evaluation of students and teachers, and points out the direction for the next research work.

\section{ACKNOWLEDGEMENTS}

Setting-sail Scientific Research Program for New Faculty of Beijing International Studies University (No.: KYQH20A035).

Quanyue Wang, https://orcid.org/0000-0002-1664-6645

Sisi Zhang, https://orcid.org/0000-0001-9655-8161

Weichao Liu, https://orcid.org/0000-0001-9086-1370 


\section{REFERENCES}

[1] Reynolds, B.-L.: Evidence for the task-induced involvement construct in incidental vocabulary acquisition through digital gaming, The Language Learning Journal, 45(4), 2017, 466-484. https://doi.org/10.1080/09571736.2014.938243

[2] Zou, D.; Huang, Y.; Xie, H.: Digital game-based vocabulary learning: where are we and where are we going?, Computer Assisted Language Learning, 1(2), 2019, 1-27. https://doi.org/10.1080/09588221.2019.1640745

[3] Lyu, G.; Chu, X.; Xue, D.: Product modeling from knowledge, distributed computing and lifecycle perspectives: A literature review, Computers in Industry, 8(4), 2017, 1-13. https://doi.org/10.1016/j.compind.2016.11.001

[4] Li, R.; Meng, Z.; Tian, M.: Modelling Chinese EFL learners' flow experiences in digital gamebased vocabulary learning: the roles of learner and contextual factors, Computer Assisted Language Learning, 1(2),2019: 1-22. https://doi.org/10.1080/09588221.2019.1619585

[5] Sun, Y.; Liu, N.; Shang, J.: Sustainable utilization of water resources in China: A system dynamics model, Journal of cleaner production, 14(2), 2017, 613-625. https://doi.org/10.1016/j.jclepro.2016.07.110

[6] Wu, Y.-C.; Yin, F.; Liu, C.-L.: Improving handwritten Chinese text recognition using neural network language models and convolutional neural network shape models, Pattern Recognition, 6(5), 2017, 251-264. https://doi.org/10.1016/j.patcog.2016.12.026

[7] Yang, Q.-F.; Chang, S.-C.; Hwang, G.-J.: Balancing cognitive complexity and gaming level: Effects of a cognitive complexity-based competition game on EFL students' English vocabulary learning performance, anxiety and behaviors, Computers \& Education, 14(8), 2020, 103-108. https://doi.org/10.1016/j.compedu.2020.103808

[8] Zhang, D.-J.; He, F.-Z.; Han, S.-H.: Quantitative optimization of interoperability during feature-based data exchange, Integrated Computer-Aided Engineering, 23(1), 2016, 31-50. https://doi.org/10.3233/ICA-150499

[9] Jianwei, W.; Zhang, J.; Yang, Q.: Modeling Design and Evaluation of Rotary Tiller Based on Multidisciplinary Optimization, International Conference on Mechanical Design, 2(2), 2019, 669-685. https://doi.org/10.1007/978-981-32-9941-2 55

[10] Ravi, S.; Maloji, S.; Polurie, V.-V.-K.; Eepuri, K.-K.: Sign language recognition with multi feature fusion and ann classifier, Turkish Journal of Electrical Engineering and Computer ences, 26(6), 2018, 2872-2886. https://doi.org/10.3906/elk-1711-139 